\title{
PHYSICOCHEMICAL STUDIES ON SOME HYDRAZONE COMPLEXES
}

HAMDY A. HAMMAD

Chemistry Department, Faculty of Science, Al-Azhar University, Nasr City, Cairo 11884, Egypt

\begin{abstract}
Some hydrazones and their complexes were prepared in order to study their molecular structures. The hydrazones were prepared by condensing p-aminobenzoic acid hydrazide with some aldehydes. The of $\mathrm{Cu}(\mathrm{II}), \mathrm{Ni}(\mathrm{II})$ and $\mathrm{Co}(\mathrm{II})$ were prepared and characterized by elemental analysis, magnetic, electronic and IR methods.
\end{abstract}

\section{Introduction}

Acid hydrazides and hydrazones received a considerable attention as antibacterial substances ${ }^{(1)}$, catalytic $^{(2)}$ and versatile chelating agents ${ }^{(3,4)}$. A survey of literature reveals that a considerable amount of work on their transition metal complexes have been carried out ${ }^{(5-16)}$.

The present paper reports the results of synthesis, characterization and of $\mathrm{Cu}(\mathrm{II})$, $\mathrm{Ni}(\mathrm{II})$ and $\mathrm{Co}(\mathrm{II})$ acetate with P-aminobenzoyl salicyl hydrazone (ABSH) and Paminobenzoyl naphthyl hydrazone (ABNH), in order to investigate their molecular structures.

\section{Experimental}

All chemicals used are of pure grade p-amino-benzoic acid hydrazide was prepared by previous method ${ }^{(17)}$. ABSH and ABNH were prepared by condensing paminobenzoic acid hydrazide with salicylaldehyde and o-hydroxynaphthaldehyde. The products were crystallized from ethanol. Metal complexes were prepared by refluxing aqueous ethanolic solution of the ligand and metal acetate in the appropriate ratio (1:1) for two hours. After cooling the complexes were filtered off, washed with ethanol and dried in vacuo. The complexes were chemically analysed for $\mathrm{C}, \mathrm{H}, \mathrm{N}$ and metals and the results are given in Table (1). Magnetic susceptibility measurements were obtained at room temperature using Gouy method. Electronic spectra were recorded as $\mathrm{NaCl}$ disks, where small amount of complexes were mixed with $0.1 \mathrm{gm} \mathrm{NaCl}$. The electronic spectra were recorded using Perkin-Elmer $\lambda_{20}$ spectrophotometer. Infrared spectra of the complexes were measured using PerkinElmer Spectrum 1 spectrophotometer.

\section{Results and Discussion}


The prepared complexes were analysed and their elemental analysis (Table 1) indicate that these complexes possess 1:1 (metal : ligand) stoichiometry. The IR spectra are listed in Table (II). The IR spectra of the ligands indicate the presence of amide I ( $v \mathrm{C}=\mathrm{O})$, azomethine $(v \mathrm{C}=\mathrm{N})$, amide II $(\delta \mathrm{NH})$, occurring at about 1710 , 1620 and $1600 \mathrm{~cm}^{-1}$ respectively, while $\delta \mathrm{OH}$ and $v \mathrm{CO}$ bands appeared at 1350 and $1170 \mathrm{~cm}^{-1}$. The comparison between the spectra of the ligands and the spectra of complexes indicate that $v \mathrm{C}=\mathrm{O}$ is shifted to higher frequency in the spectra of complexes. It is thus believed that $\mathrm{C}=\mathrm{O}$ group does not participate in complex formation. The inspection of the spectra of complexes reveals remarkable shifts to lower frequency in the azomethine, amide II and vCO bands upon complexation. Furthermore, the absorption bands at about $1355 \mathrm{~cm}^{-1}$, attributable to $\delta \mathrm{OH}$ in the free ligands, disappeared in the spectra of these complexes. These changes in the group vibrations of the azomethine and $\mathrm{CO}$ absorption, beside the disappearance of $\delta \mathrm{OH}$ mode are consistent with the coordination through the phenolic oxygen and azomethine nitrogen. The changes in the position and intensity of the broade absorption bands at about $3400 \mathrm{~cm}^{-1}$, in addition to weak absorption peaks at about 780 and $510 \mathrm{~cm}^{-1}$ in the IR spectra of these complexes, indicate the presence of coordinated water molecules ${ }^{(18)}$. In addition the spectra of complexes contain strong absorption bands at about 1600 and $1540 \mathrm{~cm}^{-1}$, characteristic of the coordinated

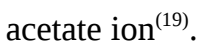

Magnetic susceptibility measurements showed that all complexes are paramagnetic. The obtained values of the effective magnetic moment $\left(\mu_{\mathrm{eff}}\right)$ indicate that the complexes have octahedral symmetry ${ }^{(20)}$. The data are listed in Table (3). The electronic spectra of the ligands and their complexes were recorded as $\mathrm{NaCl}$ disks. The colour and electronic spectral data for complexes are given in Table (3). The data indicated that these complexes are octahedrally coordinated ${ }^{(21)}$ in agreement with the conclusions from the magnetic susceptibility measurements. On the basis of preceding discussion, the suggested structure of the metal chelates under investigation is shown in Fig. (1).

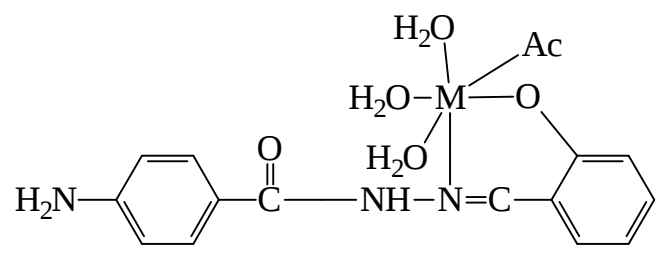

Figure (1): Proposed structure for metal-chelates. 
PHYSICOCHEMICAL STUDIES ON SOME HYDRAZONE ....

Table (1): Physical and Analytical data of ABSH, ABNH and their complexes.

\begin{tabular}{|c|c|c|c|c|c|c|c|}
\hline \multirow[t]{2}{*}{ Compound } & \multirow[t]{2}{*}{ m.p. } & \multirow[t]{2}{*}{ M wt } & \multirow[t]{2}{*}{ Molecular Formula } & \multicolumn{4}{|c|}{$\begin{array}{l}\text { Chemical Analysis } \\
\text { Calculated/Found }\end{array}$} \\
\hline & & & & $\mathrm{C}$ & $\mathrm{H}$ & $\mathrm{N}$ & M \\
\hline \multirow[t]{2}{*}{ ABSH } & \multirow[t]{2}{*}{82} & \multirow[t]{2}{*}{254} & \multirow{2}{*}{$\mathrm{C}_{14} \mathrm{H}_{12} \mathrm{~N}_{3} \mathrm{O}_{2}$} & 66.1 & 4.7 & 16.5 & - \\
\hline & & & & 65.8 & 4.4 & 16.4 & - \\
\hline \multirow[t]{2}{*}{$\mathrm{Cu}^{2+}-\mathrm{ABSH}$} & \multirow[t]{2}{*}{162} & \multirow[t]{2}{*}{429} & \multirow[t]{2}{*}{$\mathrm{C}_{14} \mathrm{H}_{11} \mathrm{~N}_{3} \mathrm{O}_{2} \mathrm{CuAc} 3 \mathrm{H}_{2} \mathrm{O}$} & 44.7 & 4.6 & 9.7 & 14.6 \\
\hline & & & & 44.5 & 4.2 & 9.3 & 14.3 \\
\hline \multirow[t]{2}{*}{$\mathrm{Ni}^{2+}-\mathrm{ABSH}$} & \multirow[t]{2}{*}{$<300$} & \multirow[t]{2}{*}{424} & \multirow[t]{2}{*}{$\mathrm{C}_{14} \mathrm{H}_{11} \mathrm{~N}_{3} \mathrm{O}_{2} \mathrm{NiAc} \mathrm{H}_{2} \mathrm{O}$} & 45.2 & 4.7 & 9.9 & 13.6 \\
\hline & & & & 44.0 & 4.5 & 9.7 & 13.2 \\
\hline \multirow[t]{2}{*}{$\mathrm{Co}^{2+}-\mathrm{ABSH}$} & \multirow[t]{2}{*}{270} & \multirow[t]{2}{*}{424} & \multirow[t]{2}{*}{$\mathrm{C}_{14} \mathrm{H}_{11} \mathrm{~N}_{3} \mathrm{O}_{2} \mathrm{CoAc}_{3} \mathrm{H}_{2} \mathrm{O}$} & 45.2 & 4.7 & 9.9 & 13.6 \\
\hline & & & & 44.1 & 4.6 & 9.6 & 13.4 \\
\hline \multirow[t]{2}{*}{$\mathrm{ABNH}$} & \multirow[t]{2}{*}{130} & \multirow[t]{2}{*}{304} & \multirow[t]{2}{*}{$\mathrm{C}_{18} \mathrm{H}_{14} \mathrm{~N}_{3} \mathrm{O}_{2}$} & 71.0 & 4.6 & 13.8 & - \\
\hline & & & & 70.8 & 4.3 & 13.6 & - \\
\hline \multirow[t]{2}{*}{$\mathrm{Cu}^{2+}-\mathrm{ABNH}$} & \multirow[t]{2}{*}{208} & \multirow[t]{2}{*}{479} & \multirow[t]{2}{*}{$\mathrm{C}_{18} \mathrm{H}_{13} \mathrm{~N}_{3} \mathrm{O}_{2} \mathrm{CuAc}_{3} \mathrm{H}_{2} \mathrm{O}$} & 50.1 & 4.5 & 8.7 & 13.1 \\
\hline & & & & 49.7 & 4.3 & 8.5 & 12.9 \\
\hline \multirow[t]{2}{*}{$\mathrm{Ni}^{2+}-\mathrm{ABNH}$} & \multirow[t]{2}{*}{$<300$} & \multirow[t]{2}{*}{474} & \multirow[t]{2}{*}{$\mathrm{C}_{18} \mathrm{H}_{13} \mathrm{~N}_{3} \mathrm{O}_{2} \mathrm{NiAc} \mathrm{H}_{2} \mathrm{O}$} & 50.6 & 4.6 & 8.8 & 12.2 \\
\hline & & & & 50.2 & 4.5 & 8.6 & 12.0 \\
\hline \multirow[t]{2}{*}{$\mathrm{Co}^{2+}-\mathrm{ABNH}$} & \multirow[t]{2}{*}{244} & \multirow[t]{2}{*}{474} & $\mathrm{C}_{18} \mathrm{H}_{13} \mathrm{~N}_{3} \mathrm{O}_{2} \mathrm{CoAc}_{3} \mathrm{H}_{2} \mathrm{O}$ & 50.6 & 4.6 & 8.8 & 12.2 \\
\hline & & & & 50.3 & 4.4 & 8.5 & 11.9 \\
\hline
\end{tabular}

Table (2): Infrared characteristic frequency vibrations of $\mathrm{ABSH}, \mathrm{ABNH}$ and their complexes.

\begin{tabular}{|c|c|c|c|c|c|c|c|c|}
\hline & ABSH & $\begin{array}{c}\mathrm{Cu}^{2+}- \\
\mathrm{ABSH} \\
\end{array}$ & $\begin{array}{l}\mathrm{Ni}^{2+}- \\
\mathrm{ABSH} \\
\end{array}$ & $\begin{array}{l}\mathrm{Co}^{2+}- \\
\mathrm{ABSH} \\
\end{array}$ & ABNH & $\begin{array}{c}\mathrm{Cu}^{2+}- \\
\mathrm{ABNH} \\
\end{array}$ & $\begin{array}{c}\mathrm{Ni}^{2+}- \\
\mathrm{ABNH} \\
\end{array}$ & $\begin{array}{c}\mathrm{Co}^{2+}- \\
\mathrm{ABNH} \\
\end{array}$ \\
\hline$v \mathrm{OH}$ & 3400 w.br. & 3422 m.br. & $\begin{array}{c}3450 \text { m.br. } \\
3376\end{array}$ & 3392 s.br & 33903m.br & 3464 m.br & 3400 m.br & 3470 m.br \\
\hline $\begin{array}{r}v \mathrm{NH}+ \\
\mathrm{NH}_{2}\end{array}$ & 3060 w.br. & 3058 w.br. & 3025 w.br & 3025 w.br & 3050 w.br & 3048 w.br & 3050 w.br & 3040 w.br \\
\hline $\begin{array}{l}\text { amid I } \\
(v=0)\end{array}$ & $1706 \mathrm{~s}$ & 1712 vs & 1708 vs & $1714 \mathrm{~m}$ & $1710 \mathrm{~s}$ & $1716 \mathrm{~s}$ & 1714 vs & $1716 \mathrm{~s}$ \\
\hline $\begin{array}{r}v \mathrm{C}=\mathrm{O} \\
\text { (acetate) }\end{array}$ & - & 1602 vs & 1602 vs & 1602 vs & - & $1598 \mathrm{~s}$ & $1598 \mathrm{~s}$ & $1600 \mathrm{~m}$ \\
\hline$\delta \mathrm{NH}$ & $1600 \mathrm{~s}$ & 1588 vs & 1575 sh & $1575 \mathrm{sh}$ & $1594 \mathrm{~s}$ & $1578 \mathrm{~s}$ & 1582 vs & $1568 \mathrm{~s}$ \\
\hline $\begin{array}{r}v \mathrm{C}=\mathrm{O} \\
\text { (acetate) }\end{array}$ & - & 1536 vs & $1542 \mathrm{~s}$ & 1542 vs & - & 1540 vs & 1540 vs & $1532 \mathrm{~s}$ \\
\hline$\delta \mathrm{OH}$ & $1360 \mathrm{~m}$ & - & - & - & $1350 \mathrm{~m}$ & 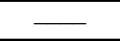 & - & - \\
\hline$v \mathrm{CO}$ & $1166 \mathrm{~s}$ & 1152 vs & 1152 vs & 1152 & $1172 \mathrm{~m}$ & $1164 \mathrm{~s}$ & $1162 \mathrm{~s}$ & $1164 \mathrm{~s}$ \\
\hline $\begin{array}{r}\mathrm{CH}_{3} \\
\text { acetate }\end{array}$ & - & $1330 \mathrm{~m}$ & $1340 \mathrm{w}$ & 1334 & - & $1344 \mathrm{~m}$ & $1344 \mathrm{~s}$ & $1340 \mathrm{sh}$ \\
\hline $\mathrm{H}_{2} \mathrm{O}$ & - & $796 \mathrm{w}$ & $800 \mathrm{sh}$ & 792 & - & $776 \mathrm{w}$ & $776 \mathrm{w}$ & $770 \mathrm{~m}$ \\
\hline $\mathrm{H}_{2} \mathrm{O}$ & - & $482 \mathrm{w}$ & $516 \mathrm{w}$ & 490 & - & $526 \mathrm{w}$ & $532 \mathrm{w}$ & $502 \mathrm{~m}$ \\
\hline$v \mathrm{M}-\mathrm{O}$ & - & $458 \mathrm{w}$ & $545 \mathrm{w}$ & 458 & - & $498 \mathrm{w}$ & $448 \mathrm{~m}$ & $542 \mathrm{w}$ \\
\hline
\end{tabular}


Table (3a): Colour and electronic spectral data of ABSH and ABNH.

\begin{tabular}{|c|c|c|c|}
\hline Compound & Colour & Peak position $\mathrm{cm}^{-1}$ & Assignment \\
\hline ABSH & Light yellow & $20,000 \& 27,397$ & \\
\hline ABNH & Orange & $19,607 \& 22,222 \& 31,250$ & $\mathrm{n} \rightarrow \pi^{*}$ and $\pi \rightarrow \pi^{*}$ \\
\hline
\end{tabular}

Table (3b): Colour and electronic spectral data of copper complexes.

\begin{tabular}{|c|c|c|c|}
\hline Compound & Colour & $\mu_{\text {eff }}$ & ${ }^{2} \mathrm{~T}_{2 \mathrm{~g}} \leftarrow{ }^{2} \mathrm{E}_{\mathrm{g}} \mathrm{Cm}^{-1}$ \\
\hline $\mathrm{Cu}^{2+}-\mathrm{ABSH}$ & Dark brown & 1.89 & 13,333 \\
\hline $\mathrm{Cu}^{2+}-\mathrm{ABNH}$ & Brown & 1.87 & 13,227 \\
\hline
\end{tabular}

Table (3c): Colour, $\mu_{\text {eff }}$ and electronic spectral data of nickel complexes.

\begin{tabular}{|c|c|c|c|c|}
\hline Compound & Colour & $\mu_{\text {eff }} B M$ & ${ }^{3} \mathrm{~T}_{1 \mathrm{~g}}+\mathrm{v}_{2}-{ }^{3} \mathrm{~A}_{2 \mathrm{~g}}$ & ${ }^{3} \mathrm{~T}_{1 \mathrm{~g}(\mathrm{p})} \quad{ }_{-1}{ }^{v_{3}}-{ }^{3} \mathrm{~A}_{2 \mathrm{~g}}$ \\
\hline $\mathrm{Ni}^{2+}-\mathrm{ABSH}$ & Green & 3.25 & 18,867 & 24,396 \\
\hline $\mathrm{Ni}^{2+}-\mathrm{ABNH}$ & Green & 3.15 & 15,384 & 27,027 \\
\hline
\end{tabular}

Table (3d): Colour, $\mu_{\text {eff }}$ and electronic spectral data of cobalt complexes.

\begin{tabular}{|c|c|c|c|c|}
\hline Compound & Colour & $\mu_{\text {eff }}$ & ${ }^{4} \mathrm{~T}_{2 \mathrm{~g}}+{ }^{v_{2}}-{ }^{4} \mathrm{~T}_{1 \mathrm{~g}}$ & ${ }^{4} \mathrm{~T}_{1 \mathrm{~g}(\mathrm{p})}+{ }^{v_{3}-}{ }^{4} \mathrm{~T}_{1 \mathrm{~g}}$ \\
\hline $\mathrm{Co}^{2+}-\mathrm{ABSH}$ & Brown & 4.87 & 18,348 & 24,641 \\
\hline $\mathrm{Co}^{2+}-\mathrm{ABNH}$ & $\begin{array}{c}\text { Reddish } \\
\text { brown }\end{array}$ & 4.62 & 16,666 & 23,809 \\
\hline
\end{tabular}

\section{References}

1. M. KATAYL AND Y. DUTT, Talanta, 22, 151, (1975).

2. D.W. STEPHEN AND T.A. WARK, Inorg. Chem., 26, 363 (1987).

3. B.K. MOHAPATRA, S. GURU AND C.D. RAO, J. Inorg. Nucl. Chem. 42, 1195 (1980).

4. M. CEBA, J.J. NEVADA AND A.E. MANSILLA, Talanta 28, 134 (1981).

5. K.K. NARANG AND A.A. AGGARWAL, Inorg. Chimica Acta, 9, 137 (1974).

6. D.K. RASTOGI, S.K. SAHNI, V.B. RANA AND S.K. DUA, Trans. Met. Chem. 3, 56 (1978).

7. D.K. RASTOGI, S.K. SAHNI, V.B. RANA AND S.K. DUA, Indi. J. Chem. 15A, 86 (1978).

8. D.K. RASTOGI, S.K. DUA, V.B. RANA AND S.K. SAHNI, J. Inorg. Nucl. Chem. 40, 1323 (1978). 
9. R.L. DUTTA AND A.K. SARKAR, ibid, 43, 57 (1981).

10. AHMED A. SHABANA, KAMEL A. EL-MANAKLY AND HAMDY A. HAMMAD, Canad. J. Appl. Spec. 39, 6, (1994).

11. M.A. KHALED, HAMDY A. HAMMAD, ATEF M. ABDALLA, AND KAMEL A. El-Manakhly ibid 40, 71 (1995).

12. KAMEL A. EL-MANAKHLY, AHMED A. SHABANA AND H.A. HAMMAD, J. Ind. Chem. Soc. 74, 715 (1997).

13. R.A. LAL AND A. KUMAR, Ind. J. Chem. 38A, 839, (1999).

14. Arindam Rana, Pupam Dinda, Sktiprosad and Alxenander J. Blake, Polyhderon, 22, 3075, (2003).

15. MOHAMMED REZA Ganjalia, Parsia Matloo, Maryam G. Horbani and Massoud Salvati - Nissar, Bull Kore. Soc. (2005), 26, 38.

16. HAMDY A. HAMMAD AND GAMAL M. EL-SHERBINY, Al-Azhar Bull. Sci., 18, 179, (2007).

17. TH. CURTIUS J. Parkt. Chem. 81, 543 (1910), 95, 336 (1917).

18. K. NAKAMOTO "Infrared Spectra of Inorganic and Coordination Compounds". Wiely, Intersience, New York, (1970).

19. K. NAKAMATO et al., J. Am. Chem. Soc. 79, 4904 (1957).

20. R.S. DRAGO “Physical Methods in Inorganic Chemistry”. East-West Eddition (1978).

21. A.B. LEVER “Inorganic Electronic Spectroscopy”. Elsevier, Amesterdam (1968). 